11.8 in the control group (Mayou et al, 2000). This may indicate that the trauma of meeting a debriefer for 1 hour was comparable to the trauma of the traffic accident itself.

If the findings of Mayou et al are valid, it shows a tremendous potential for psychological intervention. However, we are still not convinced that a 1 -hour psychological intervention unintentionally can do so much harm.

Bisson, J. I., Jenkins, P. L., Alexander, J., et al (1997) Randomised controlled trial of psychological debriefing for victims of acute burn trauma. British journal of Psychiatry, I7I, 78-8I.

Mayou, R. A., Ehlers, A. \& Hobbs, M. (2000)

Psychological debriefing for road traffic accident victims. Three-year follow-up of a randomised controlled trial. British Journal of Psychiatry, 176, 589-593.

Rose, S., Brewin, C. R., Andrews, B., et al (1999) A randomized controlled trial of individual psychological debriefing for victims of violent crime. Psychological Medicine, 29, 793-799.

Ø. Ekeberg, E. Hem Department of Behavioural Sciences in Medicine, University of Oslo, PO Box IIII Blindern, N-0317 Oslo, Norway

\section{Diazepam in the treatment of GHB dependence}

We read with great interest the recent case reported by Price (2000) concerning the utility of diazepam in suppressing gammahydroxybutyrate (GHB) dependence and related withdrawal symptoms. Before describing the case Price states, "I believe this to be the first reported case of in-patient detoxification". However, the first report on the utility of the same doses of diazepam in treating GHB withdrawal syndrome was published a year earlier by our group in a patient taking about $181 \mathrm{~g} /$ day GHB for 4 months, for its euphoric and anxiolytic effects (Addolorato et al, 1999). On discontinuation of GHB, the patient showed a withdrawal syndrome consisting of high anxiety levels, tremor, sweating, tachycardia and nausea. Complete disappearance of drug withdrawal symptoms was achieved within 2 hours in the first day of treatment with diazepam $20 \mathrm{mg}$ orally administered, and the patient was treated with the same dose of diazepam for another 6 days. After suspension of the diazepam, the symptoms did not recur. Also in this case, the detoxification programme was safe. We are very pleased to know that our findings are in perfect agreement with that of Price; since several cases of GHB misuse and dependence have been described in recent years (see Addolorato et al, 2000), we think that these reported experiences, as well as the recent case described by Hutto et al (2000) about the utility of chloral hydrate, could be of clinical relevance, particularly considering the difficult management of these patients.

Addolorato, G., Caputo, F., Capristo, E., et al (1999) A case of gamma-hydroxybutyric acid withdrawal syndrome during alcohol addiction treatment: utility of diazepam administration. Clinical Neuropharmacology, 22 $60-62$.

_, _, _, et al (2000) Gamma-hydroxybutyric acid efficacy, potential abuse and dependence in the treatment of alcohol addiction. Alcohol, 20, 17-217-222.

Hutto, B., Faichild, A. \& Bright, R. (2000) Gammahydroxybutyrate withdrawal and chloral hydrate. American Journal of Psychiatry, 157, 1706.

Price, G. (2000) In-patient detoxification after GHB dependence (letter). British Journal of Psychiatry, 177, 181.

G. Addolorato, F. Caputo, E. Capristo,

G. Gasbarrini Institute of Internal Medicine, Catholic University of Rome, Largo A. Gemelli 8, 00168 Rome, Italy

\section{Seasonality of suicides}

We read with interest Yip et al's (2000) report on the decrease or disappearance in seasonal variation of suicides in the $1980 \mathrm{~s}$ and 1990s in England and Wales. The authors have suggested a similar trend in other countries (Ho et al, 1997; Yip et al, 1998). This paper inspired us to explore seasonality of suicide in Japan, where national data on monthly suicides are collected and published every year by the Ministry of Welfare. We examined monthly data by gender for the 15 -year period 1982-1996. A daily mean suicide incidence was calculated for each month. Harmonic analysis (Pocock, 1974) was applied to identify seasonal components in the variation of monthly suicides.

A total of 332651 suicides (215 686 male, 116965 female) were identified for the period (mean suicide rate 25.8 and 13.2 per 100000 for men and women, respectively). During the entire period, the age distribution of suicide rates, which generally increases with age, and popular methods of suicides (approximately 60\% violent, nearly $35 \%$ non-violent, remainder unclassifiable or method not known) were generally unchanged. Regardless of gender, mean daily suicide incidence by month demonstrated a clear bimodal distribution, with a largest peak in April and a smaller peak in September. Harmonic analyses revealed that over $65 \%(66.7 \%$ for men and $66.2 \%$ for women) of the total variance in suicides was explained by the seasonal component, where one-cycle and two-cycle components illustrate the majority of total seasonal harmonics (one-cycle: $64.3 \%$ for men and $69.1 \%$ for women; two-cycle: $32.9 \%$ for men and $28.7 \%$ for women). No gender difference was found in the results from the harmonic analyses.

Unfortunately, no data on monthly suicides by age, by method or by place are available from the Japanese Government, which complicates further analyses regarding the backgrounds of such a marked seasonality in suicides. It may be noted that the school and business year begins in April in Japan, which may affect the seasonality of suicides, at least the largest peak of suicides in April. Overall, the results of our analyses indicate limitations in generalising from Yip et al's conclusions and suggest a wide variability in seasonality of suicide in different areas.

Ho, T. P., Chao, A. \& Yip, P. S. F. (1997) Seasona variation in suicides re-examined: no sex difference in Hong Kong and Taiwan. Acta Psychiatrica Scandinavica, 95, 25-31.

Pocock, S. J. (1974) Harmonic analysis applied to seasonal variations in sickness absence. Applied Statistics, 23, $103-120$

Yip, P. S. F., Chao, A. \& Ho, T. P. (1998) A reexamination of seasonal variation in suicides in Australia and New Zealand. Journal of Affective Disorders, 47. $|4|-150$.

\& Chiu, C.W. F. (2000) Seasonal variation in suicides: diminished or vanished. Experience from England and Wales, 1982-1996. British Journal of Psychiatry, 177, 366-369.

T. Sato Psychiatrische Klinik and Poliklinik, LMU München, Nussbraumstrasse 7, 80336 München, Germany

Reflecting on the diminished seasonal variation in suicides reported by Yip et al (2000), I was mindful of the recent extreme weather conditions - gales, floods, tornadoes - and the absence of 'Novemberness' - trees retaining their leaves, moderate temperatures, the absence of frost. Additionally in recent years we have been spared cold winters and lamented the loss of any recognisable British summertime. Therefore, one might wonder whether the disappearance of seasonal variation in suicide mirrors the 
disappearance of familiar markers of the seasons. In the absence of hard data, perhaps global warming rather than global communication (e-mail, telephone, etc.) underlies the observed trend. In future maybe other changes in traditionally seasonally linked illness patterns may be seen (e.g. in seasonal affective disorder) and this may also account for the current excess of patients with mania on our local admission ward - they seem somehow to have missed the expected springtime peak.

Reduced suicide rates among the divorced and widowed may be linked to societal changes such as the greater acceptability of single status.

We may expect human cycles (illness, behaviour) to change in line with adaptation of society and the eco- and planetary system.

But for now let us hope that there is no direct causal link between psychiatric morbidity and rainfall $(\mathrm{Ohl} \&$ Tapsell, 2000).

Ohl, A. \& Tapsell, S. (2000) Flooding and human health. British Medical Journal, 32I, I167-1168.

Yip, P. S. F., Chao, A. \& Chiu, C. W. F. (2000) Seasona variation in suicides: diminished or vanished. Experience from England and Wales, 1982-1996. British Journal of Psychiatry, I77, 366-369.

S. Smith Hillcrest Unit,Worcestershire Community and Mental Health NHS Trust, Quinneys Lane, Redditch, Worcestershire B98 7WG

\section{Remember the depressed vegetarian}

A recent experience has raised the issue of whether prescribers consider the suitability of medication for their vegetarian patients. A 30-year-old woman was prescribed an antidepressant for a depressive disorder. She later informed us that she could not take them as they contained gelatin, which is not suitable for vegetarians. This experience raises many questions. Which antidepressants contain gelatin, are prescribers aware of this information and do prescribers routinely enquire whether their patients are vegetarians prior to prescribing antidepressant medication?

We researched commonly used antidepressants by contacting the pharmaceutical companies regarding the origin of the excipients in their antidepressants. Those investigated included fluoxetine, citalopram, paroxetine, sertraline, reboxetine, venlafaxine, amitriptyline, dothiepin, imipramine, lofepramine, trazodone and nefazodone. Of these, five antidepressants are definitely suitable for vegetarians. These are sertraline tablets, venlafaxine tablets (but not capsules), fluoxetine liquid (but not capsules or tablets), amitriptyline mixture (but not capsules or tablets) and imipramine mixture (but not tablets).

Assuming the lifetime risk of developing a depressive disorder warranting treatment is $10 \%$ and there are about 4 million vegetarians in the UK (The Vegetarian Society, Altrincham, personal communication, 2000), 400000 vegetarians may need antidepressant medication.

Hence, vegetarians are commonly likely to be prescribed antidepressants by their general practitioners or psychiatrists. It is important that enquiries be made routinely regarding whether an individual is a vegetarian prior to prescribing antidepressants.

S. Singsit, P. C. Naik Solihull Healthcare NHS Trust, Lyndon Clinic, Hobs Meadow, Solihull B92 8PW

\section{One hundred years ago}

\section{London County Asylum, Claybury, Essex (Annual Report for 1899)}

... Of insanity associated with senile decay only 10 per cent. were over 60 years of age, as against 18 per cent. during the previous year. General paralysis has been found in 11 per cent. of the male admissions and in nearly 4 per cent. of the female. "There has been a high percentage of insanity from alcohol, and more than double the number of women than men have been admitted suffering from mania a poth." It is also ascertained that women relapse into insanity from alcohol and are re-admitted with far greater frequency than men. Their weakened inhibition appears to be unable to withstand the slightest temptation, and Dr. Jones points out that the best treatment for such cases is that of long detention in inebriate homes, which naturally cannot apply to asylums from which patients are discharged when mentally fit. Previous attacks and hereditary influences were ascertained to be the most probable cause of insanity in 34 per cent. of the admissions. Several patients who were admitted had delusions that they were "hounded by Kruger's relatives" and that "Spion Kop" was hissed into their ears.

\section{REFERENCE}

Lancet, 22 December 1900, 1831.

Researched by Henry Rollin, Emeritus Consultant Psychiatrist, Horton Hospital, Epsom, Surrey 